\title{
On the fine structure of magnetic fields in sunspot penumbrae (Research Note)
}

\author{
J. H. Thomas ${ }^{1}$, N. O. Weiss ${ }^{2}$, S. M. Tobias ${ }^{3}$, and N. H. Brummell ${ }^{4}$ \\ 1 Department of Physics and Astronomy and Department of Mechanical Engineering, University of Rochester, Rochester, \\ NY14627-0171, USA \\ e-mail: thomas@me.rochester.edu \\ 2 Department of Applied Mathematics and Theoretical Physics, University of Cambridge, Cambridge CB3 0WA, UK \\ e-mail: now@damtp.cam.ac.uk \\ 3 Department of Applied Mathematics, University of Leeds, Leeds LS2 9JT, UK \\ e-mail: smt@maths. leeds.ac.uk \\ 4 JILA and Department of Astrophysical and Planetary Sciences, University of Colorado, Boulder, CO 80309-0440, USA \\ e-mail: brummell@solarz.colorado.edu
}

Received 3 February 2006 / Accepted 16 March 2006

ABSTRACT

\begin{abstract}
Recent observations have revealed the interlocking-comb structure of the magnetic field in the outer penumbra of a sunspot. We have argued that this structure owes its origin in part to downward pumping of magnetic flux by vigorous granular convection in the region surrounding the spot. Here we stress the difference between the inner and outer penumbra, and correct some misleading assertions in a recent paper by Spruit \& Scharmer.
\end{abstract}

Key words. sunspots - Sun: magnetic fields

Over the last fifteen years our understanding of the fine structure of magnetic and velocity fields in sunspots has been transformed by high-resolution observations, notably those obtained at La Palma on the Swedish Vacuum Solar Telescope (Title et al. 1993; Shine et al. 1994) and, more recently, with adaptive optics on the 1-m Swedish Solar Telescope (Scharmer et al. 2002; Rouppe van der Voort et al. 2004; Langhans et al. 2005; Bellot Rubio et al. 2005). These remarkable observations have revealed the interlocking-comb structure of the magnetic field in the outer penumbra, where fields that are inclined at about $50^{\circ}$ to the vertical alternate with fields that are almost horizontal and carry the Evershed outflow (Thomas \& Weiss 2004). These two families of field lines are associated with bright and dark filaments, respectively, though this correlation is imperfect.

Observations show that the more inclined fields, together with the associated flow velocities, actually plunge below the surface in the outermost $10 \%$ by radius of the spot (Bellot Rubio et al. 2004; Langhans et al. 2005). We have proposed that they are dragged down, against the influence of magnetic tension and magnetic buoyancy, by the pumping action of small-scale turbulent granular convection outside the penumbra and we have illustrated this process in highly idealized model calculations (Thomas et al. 2002; Weiss et al. 2004). If the field is constrained to be horizontal over a substantial part of the sunspot's periphery, while it is only moderately inclined over the remainder, continuity requires that the three-dimensional penumbral magnetic field should lie in interleaved sheets, intersecting the surface in radial filaments, with a difference of inclination between alternate sheets that increases from zero at the umbral boundary to about $50^{\circ}$ at the edge of the spot, as shown schematically in Fig. 1. We claim that this configuration owes its initial development to a non-axisymmetric, convectively driven, fluting instability, which has been demonstrated in simple models (Hurlburt et al. 2000; Hurlburt \& Alexander 2002; Tildesley 2003); we also conjecture that this instability is enhanced by flux pumping, giving rise to subcritical behaviour in the nonlinear regime (Tildesley \& Weiss 2004; Thomas \& Weiss 2004).

In a recent paper, Spruit \& Scharmer (2006) assert that our model is "ruled out by observations". That statement is false. Indeed, our model was developed precisely in order to explain the observations and was designed to be compatible with them. These observations show that there are two distinct families of magnetic field lines in the outer penumbra; we propose that the nearly horizontal fields in the outer $20 \%$ by radius are held down by external pumping, and that the fields between bright filaments are more steeply inclined in consequence. We are not aware of any other mechanism that can explain the geometry of these magnetic fields.

Spruit \& Scharmer (2006) attach great significance to their claim that only a small amount of flux is involved in the steeply inclined regions, which presumes the existence of a shallow penumbra, for which there is no evidence. We do not know how much flux is involved. Nor do we claim (as they assert) that there is "a significant amount of magnetic flux having opposite polarity to that of the penumbra" that emerges into the photosphere around a sunspot: the steeply inclined fields make angles of $\pm 10^{\circ}$ with the horizontal and only involve about $2 \%$ of the total flux emerging from the spot. On the other hand, the total amount of flux that protrudes from the spot below the surface depends on the thickness of the magnetic layer that is pumped downwards, which we do not know; if the layer is indeed a few Mm thick, as estimated by Weiss et al. (2004), then a very substantial amount of flux is involved. Moving magnetic features in the moat cell that surrounds the spot expose only a small sample of this flux. 


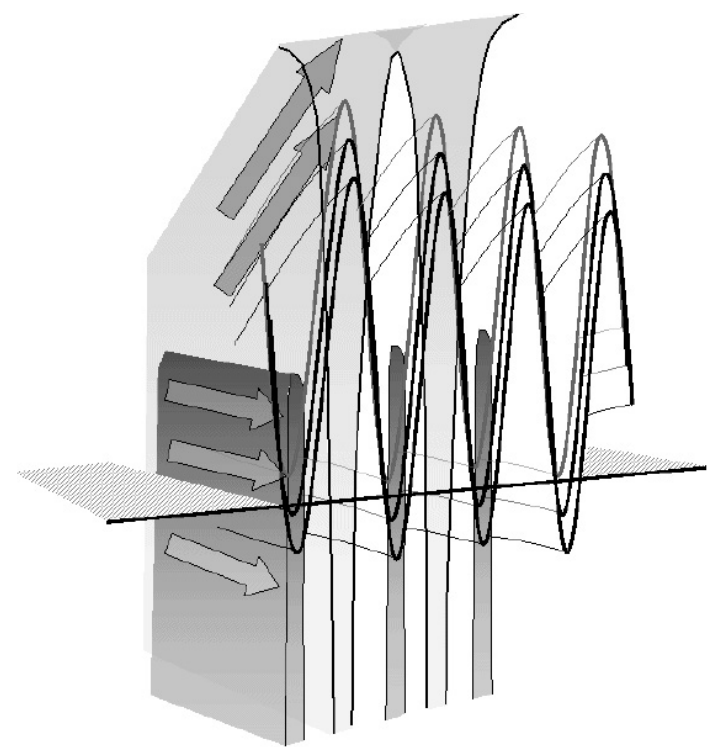

Fig. 1. Schematic diagram indicating how an initial fluting instability can develop to produce a pattern of interleaved sheets with fields inclined in different directions. The horizontal plane represents the visible surface. In the outer penumbra the less inclined fields are at an angle of about $50^{\circ}$ to the vertical, while the more inclined fields are approximately horizontal. In the outermost $10 \%$ by radius the latter actually dip downwards below the surface; we claim that this is a result of downward pumping by turbulent convection in the granulation outside the spot.

Spectropolarimetric measurements suggest that the steeply dipping fields have a limited vertical extent above the photosphere (Solanki \& Montavon 1993; Bellot Rubio et al. 2004), though their depth remains uncertain. In our picture, the sheets containing horizontal fields have a wedge-shaped cross-section that is enclosed above by the less inclined fields but extends downwards for a significant depth. This differs from the apparently shallow cross-section of the prominent dark cores within bright filaments in the inner penumbra, near the umbral boundary (Sütterlin et al. 2004). Spruit \& Scharmer (2006) propose a very appealing explanation for these cores, as dark absorption features, which is entirely consistent with our own picture of penumbral structure. It should be noted, however, that their model is two-dimensional and only applies to azimuthal variations in the inner penumbra, without attempting to reproduce radial variation or the patterns of fields and flows in the outer penumbra. We are not convinced by their insistence on fieldfree convection and a 'gappy' penumbra, which is not compatible with numerical investigations of magnetoconvection, and we shall return to this topic elsewhere.

The arguments attributed to us by Spruit \& Scharmer are distorted, and imply a lack of understanding of our model, which we hope to have clarified here. In conclusion, we emphasize the importance of distinguishing between magnetic structures in the inner penumbra and those in the outer penumbra; it is only the latter that we have attempted to describe. The new highresolution observations raise a range of challenging and fascinating questions, which can only be answered by combining precise physical arguments with detailed computation.

\section{References}

Bellot Rubio, L. R., Balthasar, H., \& Collados, M. 2004, A\&A, 427, 319

Bellot Rubio, L. R., Langhans, K., \& Schlichenmaier, R. 2005, A\&A, 443, L7

Hurlburt, N. E., \& Alexander, D. 2002, in COSPAR Colloquia Ser. 14, Solar Terrestrial Magnetic Activity and Space Environment, ed. H. Wang, \& R. Xu (Oxford: Pergamon), 19

Hurlburt, N. E., Matthews, P. C., \& Rucklidge, A. M. 2000, Sol. Phys., 192, 109

Langhans, K., Scharmer, G. B., Kiselman, D., Löfdahl, M. G., \& Berger, T. F. 2005, A\&A, 436, 1087

Rouppe van der Voort, L. H. M., Löfdahl, M. G., Kiselman, D., \& Scharmer, G. B. 2004, A\&A, 414, 717

Scharmer, G. B., Gudiksen, B. V., Kiselman, D., Löfdahl, M. G., \& Rouppe van der Voort, L. H. M. 2002, Nature, 420, 151

Shine, R. A., Title, A. M., Tarbell, T. D., et al. 1994, ApJ, 430, 413

Solanki, S. K., \& Montavon, C. A. P. 1993, A\&A, 275, 283

Spruit, H. C., \& Scharmer, G. B. 2006, A\&A, 447, 343

Sütterlin, P., Bellot Rubio, L. R., \& Schlichenmaier, R. 2004, A\&A, 424, 1049

Thomas, J. H., \& Weiss, N. O. 2004, ARA\&A, 42, 517

Thomas, J. H., Weiss, N. O., Tobias, S. M., \& Brummell, N. H. 2002, Nature, 420,390

Tildesley, M. J. 2003, MNRAS, 338, 497

Tildesley, M. J., \& Weiss, N. O. 2004, MNRAS, 350, 657

Title, A. M., Frank, Z. A., Shine, R. A., et al. 1993, ApJ, 403, 780

Weiss, N. O., Thomas, J. H., Brummell, N. H., \& Tobias, S. M. 2004, ApJ, 600, 1073 